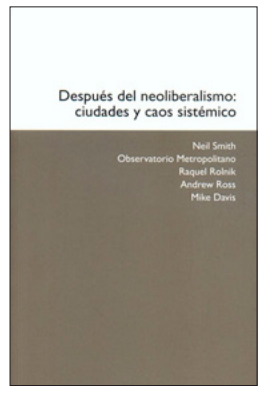

\title{
Neil Smith, Observatorio Metropolitano, Raquel Rolnik, Andrew Ross y Mike Davis. Después del neoliberalismo: ciudades y caos sistémico
}

\author{
Barcelona: Ed. Museu dÁrt Contemporani de Barcelona, \\ Universitat Autónoma de Barcelona, Colección Contra Textos, \\ 2009. 106 p.
}

Felipe Link L. ${ }^{1}$

El libro Después del neoliberalismo: ciudades y caos sistémico, es el resultado de un seminario realizado en noviembre de 2008, en el Museo de Arte Contemporáneo de Barcelona, cuyo objetivo fue discutir la vigencia y consecuencias del modelo neoliberal en las ciudades contemporáneas. La publicación resultante, recoge las intervenciones de diferentes autores en torno a los impactos socioterritoriales en ciudades como Madrid, São Paulo y Shanghai, además de un excelente capítulo introductorio de Neil Smith y una conclusión de Mike Davis.

Específicamente, el libro se desarrolla a partir de la premisa inspirada en el análisis de la modernidad de Jurgen Habermas y supone lo siguiente: el neoliberalismo ha muerto, pero sigue vigente. Es decir, se propone una reflexión acerca del futuro de las ciudades, fuertemente impactadas por las consecuencias de un neoliberalismo urbano que ha perdido fuerza, pero predominante en las últimas décadas.

\section{¿Ciudades después del neoliberalismo?}

Neil Smith plantea claramente en el primer capítulo la idea de un neoliberalismo muerto, pero vigente, en tres de los pilares fundamentales del edificio neoliberal. Esto es, la persistencia, aunque corroída de: 1) la

1 Sociólogo, Doctor en Arquitectura y Estudios Urbanos. Escuela de Sociología, Universidad Diego Portales (Chile). E-mail: felipe.link@udp.cl economía de libre mercado como la única moneda legítima de interacción social; 2) la desregulación concomitante de algunas funciones estatales y la renuncia del Estado al proyecto keynesiano de ayuda social y apoyo a la reproducción social; 3) la santidad de la propiedad privada junto con la privatización progresiva de los recursos sociales (Smith, 2009:12-13).

Este escenario, que es ciego ante la gravedad de las crisis sucesivas del sistema, y que las circunscribe territorialmente, describiéndolas metafóricamente como áreas de contagio o de activos tóxicos, sin considerar su carácter estructural, sistémico y global, se ve reforzado por la transformación de la condición urbana en la globalización. La ciudad pasa a ser una plataforma de la economía global, más allá de su condición reproductora de las economías nacionales. Por lo tanto, la relación ciudad-Estado se transforma y el desarrollo urbano se condiciona a la "amalgama de capital internacional que recodifica la ciudad".

La novedad en el análisis del autor, está en la descripción de la intensificación y densidad de estas relaciones, a la manera de una revolución urbana anticipada ya por $\mathrm{H}$. Lefebvre. Así, en un mundo eminentemente urbano "petrificado por la infusión política de un nuevo régimen de seguridad", el neoliberalismo decadente generaría caos sobre el caos, con las diferencias y particularidades propias de cada territorio.

Cada ciudad ejemplifica, a su manera, diferentes combinaciones neoliberales, así 
como diferentes escalas del problema, donde la alternativa al proyecto urbano neoliberal, estaría en el potencial productivo del caos a nivel local, al reorganizar políticamente a las ciudades con el objetivo de volver a respirar el aire de la igualdad.

A partir de esta introducción, el libro se estructura en tres capítulos que ejemplifican la realidad particular de Madrid, Shanghai y las metrópolis brasileñas, para terminar con un capítulo conclusivo de Mike Davis.

\section{Madrid. Explosión y crisis del modelo urbano}

Los autores del Observatorio Metropolitano, Eva García Pérez, Patricia Molina Costa y Emmanuel Rodríguez López, describen la explosión urbana de Madrid, como un ejemplo del impacto neoliberal en el territorio. Específicamente, expresado en una nueva centralidad con consecuencias desiguales al interior del territorio metropolitano. Según los autores, hay tres factores asociados al crecimiento explosivo de la capital, estos son:

- Consolidación de la ciudad como centro turístico, nudo logístico y mercado financiero de relevancia internacional.

- Formación de un aparato decisional compuesto por los cuarteles operativos de empresas multinacionales.

- Formación de economías de aglomeración en sectores de alto valor añadido, como los servicios a la producción.

Sin embargo, observan que "el modelo de éxito rotundo en términos macroeconómicos, se declina de una forma ambivalente si se consideran otros factores como el impacto social o los efectos en los modos de gobierno". Así, el modelo Madrid goes global se traduciría en las descripciones clásicas sobre dualización social y territorial.

En Madrid parece consolidarse un nuevo empresarialismo urbano, donde el sector público asume generalmente los riesgos y el sector privado, todos los beneficios, en una lógica de "chorreo urbano" y de subordinación a la inversión privada. Las consecuencias sociales y territoriales de estos procesos son difíciles de medir, no forman parte de la agenda pública y quedan veladas por el éxito de la "marca urbana".

\section{Confinamiento o conflagración: metrópolis brasileñas al límite}

Raquel Rolnik analiza el impacto de la crisis del neoliberalismo en las metrópolis brasileñas, describiendo los efectos de las políticas implementadas desde los años 90 en adelante.

Una de las características principales de la expansión urbana en Brasil, tiene que ver con el asentamiento de una fuerte migración rural y su concentración en "áreas de mercado" en diferentes escalas metropolitanas. Según Rolnik, "al delimitar las fronteras que separan los asentamientos regulares/formales de los irregulares/informales, el modelo de exclusión territorial que distingue la ciudad brasileña es mucho más que la mera expresión de las desigualdades sociales y de renta, en la medida en que funciona, para el circuito financiero, como una especie de engranaje de la máquina de generar valor, inflando el capital en ella invertido" (Rolnik, 2009:46). La fragmentación generada por este proceso, impone un patrón de circulación y movilidad poco sustentable y pone en cuestión al modelo neoliberal de ciudad en Brasil.

Este proceso transformó, según la autora, toda "la geografía de la pobreza urbana y de la vulnerabilidad social, lo que repercutió profundamente en la dinámica de agregación social del territorio popular y en las relaciones reales y simbólicas que este mantiene con el resto de la ciudad". (Rolnik, 2009:51). Así, la tradicional relación entre centro y periferia se desvanece ante la relación entre seguridad y violencia. La idea misma de ciudad, entendida como una condición de contacto con el otro, se ve dificultada en este escenario de fragmentación social, política y territorial.

Sin embargo, la época dorada del neoliberalismo parece estar llegando a su fin según Rolnik, dadas las permanentes crisis del modelo en todos sus niveles, desde la gestión financiera, hasta la forma de ocupación del 
territorio. Por lo tanto, solo la innovación social y el ejercicio ciudadano, aparecen como nuevas oportunidades de reforma de las relaciones sociopolíticas en la ciudad, que tiendan a generar un nuevo pacto territorial, que siguiendo a Lefevbre, privilegie el valor de uso por sobre el valor de cambio.

\section{Shanghai en el límite}

El trabajo de Andrew Ross, analiza Shanghai, entendido como un paraíso neoliberal, desde mucho antes de los procesos de globalización contemporáneos. Se trata, según el autor, de una ciudad creada por el capital extranjero. Sin embargo, las particularidades de Shanghai, tienen que ver con el fuerte apoyo estatal para enfrentar las consecuencias perversas del desarrollo urbano. En este escenario, Ross describe la apertura de la ciudad, iniciada en 1992 por Deng Xiaoping, como una "orgía de obras públicas sin precedentes". Es decir, que "el rápido desarrollo de sus espacios de libre comercio, complejos de apartamentos de la más alta categoría, campos de golf, centros comerciales y hoteles de lujo, y bolsas de cambio, estaba destinado a devolver a Shanghai su posición de la preguerra, como el primer centro comercial y financiero de la región de Asia-Pacífico" (Ross, 2009:68).

La pregunta que surge a la luz del desarrollo de Shanghai y de China en general, tiene que ver con la naturaleza de las relaciones entre el Estado y la floreciente economía privada. En esta relación, por un lado se multiplica la infraestructura, mientras por otro aumenta fuertemente la desigualdad. Ross señala el aumento del coeficiente Gini en China de 0,15 en 1978 a 0,48 en 2007 pasando a ser uno de los países con mayor desigualdad en el mundo. La dualización se observa como una amenaza creciente, ya que "en el espacio de 10 años, la clase obrera urbana en Shanghai ha pasado de ser la formada por unos ciudadanos modelo del deseado centro de la economía, a la de unos parias indeseables en los márgenes físicos de una ciudad a la que ya no pueden acceder" (Ross, 2009:73). Así, el resultado es similar al de ciudades occidentales, en el sentido de un modelo privatizador, con aumento de la desigualdad y disminución de seguridad social.
Finalmente, el autor señala que, a pesar de que el término neoliberalismo no es muy extendido en China, las similitudes con el desarrollo urbano neoliberal de ciudades como Madrid o São Paulo, parecen evidentes.

\section{¿Quién construirá el Arca de Noé? El imperativo utópico en la Era de la Catástrofe}

El artículo final del libro, a manera de conclusión y a cargo de Mike Davis, plantea un escenario de amenazas y oportunidades, de fortalezas y debilidades en la situación actual de las ciudades bajo la época neoliberal.

Por un lado, los problemas ambientales acentúan la sensación de riesgo. Según Davis, "el cambio climático producirá impactos dramáticamente desiguales en regiones y clases sociales, infligiendo los daños mayores a los países pobres con menores recursos para una adaptación significativa. Esta separación geográfica de fuentes de emisión con consecuencias ambientales mina la solidaridad proactiva." (Davis, 2009:91). Además, "la extremada velocidad del crecimiento demográfico incrementará la población urbana del mundo en cerca de 3 mil millones de personas en los próximos cuarenta años, el 90 por ciento de ellas en ciudades pobres (Davis, 2009:94), lo que contribuye a destruir los ecosistemas en todas sus dimensiones.

Por otro lado, este mismo escenario de catástrofe contiene, a la manera de una dialéctica marxista, las contradicciones propias del modelo que podrían llevar a su superación. Davis plantea un urbanismo radical, vinculado a la idea de justicia ecológica, lo que se traduciría en el privilegio del bienestar público sobre la riqueza privada. Esta reivindicación de la igualdad en la vida urbana, sería el mejor fundamento para la conservación en sentido amplio. Así, "ante las crisis crecientemente interrelacionadas de pobreza urbana y cambio climático, o bien luchamos a favor de soluciones "imposibles" o nos convertimos en cómplices de actividades que diezman a la humanidad" (Davis, 2009:103).

Finalmente, los autores del libro describen un diagnóstico real en la mayoría de las ciudades impactadas por políticas neolibera- 
les en diferentes formas y escalas. Señalan, con matices, el agotamiento del modelo predominante en las últimas décadas y cifran sus esperanzas en la organización ciudadana por un derecho a la ciudad en sentido amplio.

Las ciudades latinoamericanas viven, sin duda, un proceso similar, lo que abre oportunidades políticas de acción en un momento de bifurcación del sistema. El neoliberalismo, parece generar efectivamente un caos sistémico en el desarrollo urbano, que reconfigura la estructura social e institucional de la ciudad, priorizando, por sobre todo, el derecho a la propiedad privada. Probablemente estos principios están más vigentes que nunca, y precisamente por eso, son también los más cuestionados. Los autores entienden esta doble condición y señalan, inteligentemente, un escenario de neoliberalismo muerto, pero vigente. 